\title{
Numerical treatment of the loss of hyperbolicity of the two-layer shallow-water system
}

\author{
M. J. Castro Díaz ${ }_{, *}^{*}$ E. D. Fernández-Nieto $\stackrel{\dagger}{\dagger}$ \\ J.M. González-vida ‡ C. Parés $^{\S}$
}

\begin{abstract}
In this work, a characterization of the hyperbolicity region for the two layer shallow-water system is proposed and checked. Next, some path-conservative finite volume schemes (see [11]) that can be used even if the system is not hyperbolic are presented, but they are not in general $\mathrm{L}^{2}$ linearly stable in that case. Then, we introduce a simple but efficient strategy to enforce the hyperbolicity of the two-layer shallow-water system consisting in adding to the system an extra amount of friction at every cell in which complex eigenvalues are detected at a given time step. The implementation is performed by a predictor/corrector strategy: first a numerical scheme is applied to the unmodified two-layer system, regardless of the hyperbolic character of the system. Next, we check if the predicted cell averages are in the hyperbolic region or not. If not, the mass-fluxes are corrected by adding a quadratic friction law between layers whose coefficient is computed so that the corrected cell average is as near as possible of the boundary of the hyperbolicity region. Finally, some numerical test have been performed to assess the efficiency of the proposed strategy.
\end{abstract}

Short title : Numerical treatment of the loss of hyperbolicity of the two-layer SWS.

Keywords : Finite Volume Method, path-conservative, two-layer shallow water, complex eigenvalues.

Subject Classifications : AMS (MOS) : 65N06, 76B15, 76M20, 76N99.

\footnotetext{
*Dpto. Análisis Matemático, Universidad de Málaga, Campus Teatinos s/n, 29071 Málaga, Spain (castro@anamat.cie.uma.es)

${ }^{\dagger}$ Departamento de Matemática Aplicada I, Universidad de Sevilla. E.T.S. Arquitectura. Avda, Reina Mercedes, s/n. 41012 Sevilla, Spain (edofer@us.es).

${ }_{\ddagger}^{\ddagger}$ Dpto. de Matemática Aplicada, Universidad de Málaga, Campus de Teatinos s/n, 29071 Málaga. Spain (vida@anamat.cie.uma.es)

§Dpto. Análisis Matemático, Universidad de Málaga, Campus Teatinos s/n, 29071 Málaga, Spain (pares@anamat.cie.uma.es)
} 


\section{Introduction}

Let us consider the system of partial differential equations governing the one-dimensional flow of two superposed immiscible layers of shallow water fluids studied in [6]:

$$
\left\{\begin{array}{l}
\frac{\partial h_{1}}{\partial t}+\frac{\partial q_{1}}{\partial x}=0 \\
\frac{\partial q_{1}}{\partial t}+\frac{\partial}{\partial x}\left(\frac{q_{1}^{2}}{h_{1}}+\frac{g}{2} h_{1}^{2}\right)=-g h_{1} \frac{\partial h_{2}}{\partial x}+g h_{1} \frac{d H}{d x}+S_{1}^{f}, \\
\frac{\partial h_{2}}{\partial t}+\frac{\partial q_{2}}{\partial x}=0 \\
\frac{\partial q_{2}}{\partial t}+\frac{\partial}{\partial x}\left(\frac{q_{2}^{2}}{h_{2}}+\frac{g}{2} h_{2}^{2}\right)=-\frac{\rho_{1}}{\rho_{2}} g h_{2} \frac{\partial h_{1}}{\partial x}+g h_{2} \frac{d H}{d x}+S_{2}^{f} .
\end{array}\right.
$$

In these equations, index 1 makes reference to the upper layer and index 2 to the lower one. The fluid is assumed to occupy a straight channel with constant rectangular cross-section and constant width. The coordinate $x$ refers to the axis of the channel, $t$ is time, and $g$ is the acceleration due to gravity. $H(x)$ represents the depth function measured from a fixed level of reference (see Figure 1). Each layer is assumed to have a constant density, $\rho_{i}, i=1,2\left(\rho_{1}<\rho_{2}\right)$. The unknowns $q_{i}(x, t)$ and $h_{i}(x, t)$ represent respectively the mass-flow and the thickness of the $i$-th layer at the section of coordinate $x$ at time $t$. Finally, $S_{1}^{f}$ and $S_{2}^{f}$ represent the friction forces: $S_{1}^{f}=S_{1}^{w}+S_{1}^{i}, S_{2}^{f}=S_{2}^{b}+S_{2}^{i}$, where $S_{1}^{w}$ parameterizes the wind friction, $S_{2}^{b}$ the bottom friction, and $S_{1}^{i}, S_{2}^{i}$ the friction between the two layers. Different formula have been proposed to parameterize these friction forces. Here, the friction between layers is assumed to be given by a quadratic law of the form:

$$
S_{1}^{i}=-c\left|u_{1}-u_{2}\right|\left(u_{1}-u_{2}\right) ; \quad S_{2}^{i}=r c\left|u_{1}-u_{2}\right|\left(u_{1}-u_{2}\right),
$$

where $r=\rho_{1} / \rho_{2}$ and the coefficient $c$ may depend on the unknowns, as it is the case in the Manning law:

$$
c=c_{0} \frac{h_{1} h_{2}}{h_{2}+r h_{1}} .
$$

being

System (1.1) can be written in the following form:

$$
w_{t}+F(w)_{x}+\mathcal{B}(w) \cdot w_{x}=S(w) H_{x}+S^{f},
$$




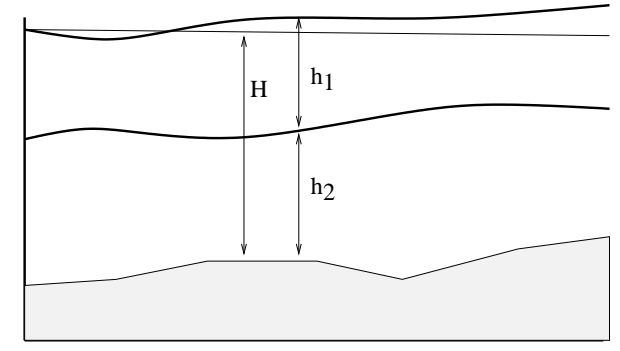

Figure 1: Two-layer sketch: unknowns $h_{1}, h_{2}$ and bathimetry function $H$.

where

$$
\begin{gathered}
w(x, t)=\left[\begin{array}{c}
h_{1}(x, t) \\
q_{1}(x, t) \\
h_{2}(x, t) \\
q_{2}(x, t)
\end{array}\right], \quad F(w)=\left[\begin{array}{c}
q_{1} \\
\frac{q_{1}^{2}}{h_{1}}+\frac{g}{2} h_{1}^{2} \\
q_{2} \\
\frac{q_{2}^{2}}{h_{2}}+\frac{g}{2} h_{2}^{2}
\end{array}\right], \quad S(w)=\left[\begin{array}{c}
0 \\
g h_{1} \\
0 \\
g h_{2}
\end{array}\right], \\
S^{f}=\left[\begin{array}{c}
0 \\
S_{1}^{f} \\
0 \\
S_{2}^{f}
\end{array}\right], \quad \mathcal{B}(w)=\left[\begin{array}{cccc}
0 & 0 & 0 & 0 \\
0 & 0 & g h_{1} & 0 \\
0 & 0 & 0 & 0 \\
g r h_{2} & 0 & 0 & 0
\end{array}\right] .
\end{gathered}
$$

The vector $w$ takes values in the set:

$$
\Omega=\left\{\left[h_{1}, q_{1}, h_{2}, q_{2}\right]^{T} \in \mathbb{R}^{4}, \quad h_{1}>0, h_{2}>0\right\} .
$$

The thickness of the layer may vanish in practical applications when one or the two layers disappear in part of the domain. Nevertheless, the numerical difficulties related to the corresponding wet-dry fronts will not be discussed here (see [4]).

It is well known that this system may lose the hyperbolic character. For $r \cong 1$ this situation occurs approximately when the following inequality is satisfied:

$$
\frac{\left(u_{1}-u_{2}\right)^{2}}{g^{\prime}\left(h_{1}+h_{2}\right)}>1
$$

This hyperbolicity loss is related to the appearance of shear instabilities that may lead, in real flows, to intense mixing of the two layers. While, in practice, this mixture partially dissipates the energy, in numerical experiments these interface disturbances may grow and overwhelm the solution.

Obviously, a simple model based on two layer of immiscible fluids is not able to simulate the mixing processes due to the development of shear instabilities: a continuously stratified model would be required. The goal of this article is to propose 
a strategy allowing us to use the two layer model to simulate flows that are essentially hyperbolic, unless for some sporadic episodes in which complex eigenvalues appear. Moreover these episodes are assumed to be very localized in space. The idea is to modify locally the model when complex eigenvalues appear in order to get rid of the related instabilities and go beyond of them by reaching again the hyperbolic character.

In previous works, it has been seen that adding some extra friction terms reduces the shear stress enforcing thus the hyperbolic character of the system (see [9], [10]). The difficulty is that, in order to avoid an excess of diffusion, this extra terms should only act locally both in time and space when the appearance of complex eigenvalues is detected. A second difficulty comes from the fact that adding extra local diffusion terms can produce nonphysical oscillatory patterns in the flow. In [10] a different strategy to overcome with this difficulty was studied that consists in neglecting the imaginary part of the complex eigenvalues.

The strategy pursued in this article consists in adding to the system an extra amount of friction to the model at every cell in which complex eigenvalues are detected at a given time level so that the updated cell average is again in the hyperbolic region. This will be done by using a predictor/corrector strategy. First, a numerical scheme is applied to the unmodified model regardless of the hyperbolic character of the system. Therefore, the numerical schemes to be used in this first stage have to be able to advance in time even in the presence of complex eigenvalues. Next, we check wether the predicted cell averages are in the hyperbolicity region or not. If not, the mass-fluxes are corrected by adding a quadratic friction law between the layers. This fictitious friction force, which is intended to simulate the loss of momentum due to the mixing processes in real flows, is semi-implicitly discretized. The key point is the calculation of the coefficient appearing in the friction law: it is calculated so that the corrected cell average is as near as possible of the boundary of the hyperbolicity region, in a sense to be determined.

Once this technique has been implemented for first order schemes and 1d models, its extension to $2 \mathrm{~d}$ models and/or high order schemes (either by means of reconstruction of states or ADER techniques) is straightforward by applying the techniques describes in [11], [2], [3], [1] ...

The organization is as follows: in next Section, we analyze the hyperbolicity regions of the system. Next, we present a family of numerical schemes based on a Roe linearization of the system which are able to advance in time even if the system is not hyperbolic. Even if these schemes are not $L^{2}$ stables when complex eigenvalues are present, as it will be seen, they will be useful to perform the prediction stage. In Section 4 the strategy to calculate the friction coefficient and to correct the predicted mass-fluxes when necessary. Finally, some numerical experiments will be shown to validate the efficiency of the strategy. 


\section{Hyperbolicity region of the two-layer shallow water system}

As the source term due to bed variations does not affect to the hyperbolic character of the system and the friction source terms enforces this character (as they tend to reduce the shear between the layers), we consider for simplicity the homogeneous system:

$$
w_{t}+F(w)_{x}+\mathcal{B}(w) \cdot w_{x}=0,
$$

in which the depth $H$ is supposed to be constant and the friction forces are neglected.

The system can be rewritten as follows:

$$
w_{t}+A(w) \cdot w_{x}=0
$$

where

$$
A(w)=\mathcal{J}(w)+\mathcal{B}(w),
$$

being $\mathcal{J}(w)$ the Jacobian matrix of $F(w)$

$$
\mathcal{J}(w)=\frac{\partial F}{\partial w}(w)
$$

The characteristic equation of the $A(w)$ is:

$$
\left(\lambda^{2}-2 u_{1} \lambda+u_{1}^{2}-g h_{1}\right)\left(\lambda^{2}-2 u_{2} \lambda+u_{2}^{2}-g h_{2}\right)=r g^{2} h_{1} h_{2},
$$

where $u_{i}=q_{i} / h_{i}$ represents the averaged velocity of the $i$-th layer, $i=1,2$.

It is easy to check that the condition under which one of the eigenvalues vanishes is:

$$
G^{2}=F_{1}^{2}+F_{2}^{2}-(1-r) F_{1}^{2} F_{2}^{2}=1,
$$

where $G$ is the so-called composite Froude number, and $F_{i}$ for $i=1,2$ are the internal Froude numbers $\left(F_{i}^{2}=\frac{u_{i}^{2}}{g^{\prime} h_{i}}\right.$, where $g^{\prime}$ is the reduced gravity, $\left.g^{\prime}=(1-r) g\right)$. When this condition is achieved at a section of coordinate $x$, the flow is said to be critical at this point and the section $x$ is called a control. When $G^{2}<1$, the flow is subcritical. Finally, when $G^{2}>1$, the flow is supercritical.

Observe that, when $r=0$, the eigenvalues are those corresponding to each layer separately. Therefore, when $r \cong 0$, the coupling terms do not affect the nature of the system in an essential manner.

In the case $r \cong 1$ (which is the situation arising in many geophysical flows) a first-order approximation of the eigenvalues was given in [13]:

$$
\begin{aligned}
& \lambda_{\text {ext }}^{ \pm} \cong \frac{u_{1} h_{1}+u_{2} h_{2}}{h_{1}+h_{2}} \pm\left(g\left(h_{1}+h_{2}\right)\right)^{\frac{1}{2}} \\
& \lambda_{\text {int }}^{ \pm} \cong \frac{u_{1} h_{2}+u_{2} h_{1}}{h_{1}+h_{2}} \pm\left(g^{\prime} \frac{h_{1} h_{2}}{\left(h_{1}+h_{2}\right)}\left[1-\frac{\left(u_{1}-u_{2}\right)^{2}}{g^{\prime}\left(h_{1}+h_{2}\right)}\right]\right)^{\frac{1}{2}} .
\end{aligned}
$$


Notice that the approximation of $\lambda_{\text {int }}^{ \pm}$become complex when (1.4) is satisfied.

Let us consider the non-dimensional variables

$$
\hat{h}_{i}=\frac{h_{i}}{\bar{H}}, \quad \hat{u}_{i}=\frac{u_{i}}{\bar{U}}
$$

where $\bar{H}$ is the depth and $U=\sqrt{g^{\prime} \bar{H}}$. In these variables the characteristic equation reduces to:

$$
\left(\hat{\lambda}^{2}-2 \hat{u}_{1} \hat{\lambda}+\hat{u}_{1}^{2}-\frac{1}{1-r} \hat{h}_{1}\right)\left(\hat{\lambda}^{2}-2 \hat{u}_{2} \hat{\lambda}+\hat{u}_{2}^{2}-\frac{1}{1-r} h_{2}\right)=\frac{r}{(1-r)^{2}} \hat{h}_{1} \hat{h}_{2},
$$

and the inequality (1.4) reads as follows:

$$
\frac{\left(\hat{u}_{1}-\hat{u}_{2}\right)^{2}}{\left(\hat{h}_{1}+\hat{h}_{2}\right)}>1 .
$$

Therefore, the hyperbolicity of the system is related to the ratio between the square of the adimensional shear and the adimensional total thickness. Figure 2 shows the hyperbolicity region in the plane of coordinates $\left(\left|\hat{u}_{1}-\hat{u}_{2}\right|, \hat{h}_{1}+\hat{h}_{2}\right)$ for different values of the parameter $r$. To obtain these plots, we have considered a number of random states $\hat{w}=\left[\hat{h}_{1}, \hat{u}_{1}, \hat{h}_{2}, \hat{u}_{2}\right]$ and the roots of the corresponding polynomial (2.7) have been numerically computed. If all the roots are real, a blue dot is drawn in the plane of coordinates $\left(\left|\hat{u}_{1}-\hat{u}_{2}\right|, \hat{h}_{1}+\hat{h}_{2}\right)$, otherwise a red dot is drawn. The curve

$$
\hat{h}_{1}+\hat{h}_{2}=\left(\hat{u}_{1}-\hat{u}_{2}\right)^{2}
$$

is also drawn in green. Note that in all the cases two hyperbolicity regions appear: a first one containing the vertical axis which is almost invariant for the different values of $r$. Moreover, it can be observed that the curve (2.8) is an excellent approximation of the boundary of this region when $r \cong 1$ (see Figures $2(\mathrm{~b})-2(\mathrm{~d})$ ), and even for smaller values (see Figuree 2(a)).

The second hyperbolic region grows for smaller values of $r$ and it corresponds, at least for $r \cong 1$, to states for which $\left(\hat{u}_{1}-\hat{u}_{2}\right)$ is big compared to $\hat{h}_{1}+\hat{h}_{2}$. Nevertheless, real stratified geophysical flows with $r \cong 1$ are located in the first region. Therefore, we can conclude that (1.4) is in practice a simple and efficient criterium to determine the hyperbolic character of the two-layer shallow-water system when the densities of the layer are close enough.

\section{$3 \quad$ Numerical schemes}

Solutions of (2.2) may develop discontinuities and, due to the non-divergence form of the equations, the notion of weak solution in the sense of distributions cannot be used. The theory introduced by Dal Maso, LeFloch, and Murat [8] is followed here to define weak solutions of (2.2) or equivalently of (1.3). This theory allows 


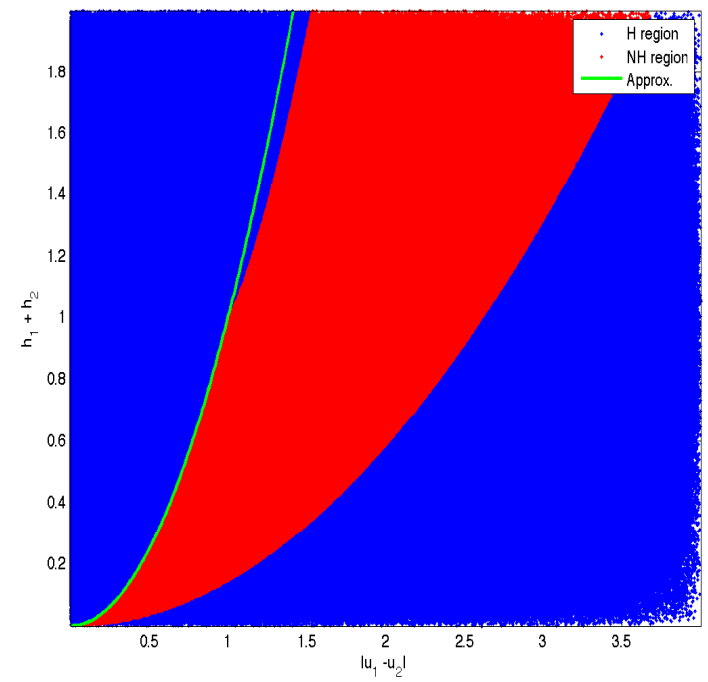

(a) Hyperbolicity region for $r=0.5$

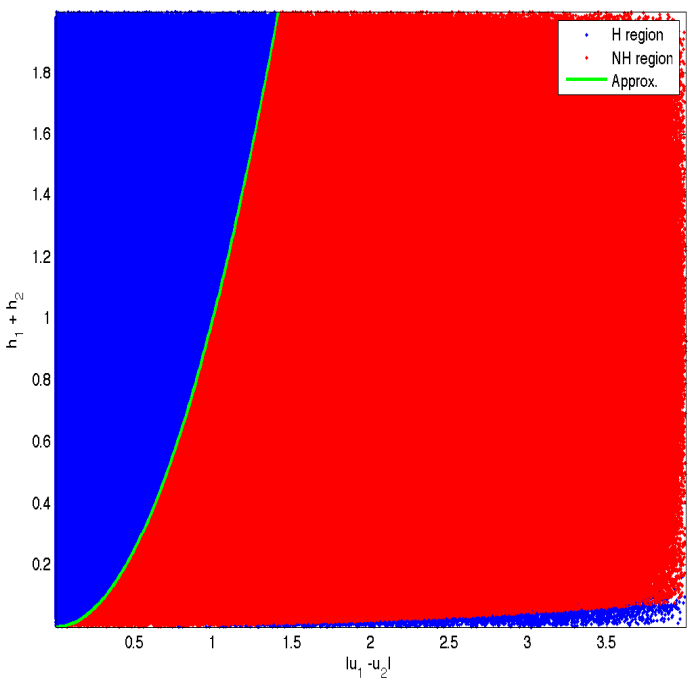

(c) Hyperbolicity region for $r=0.98$

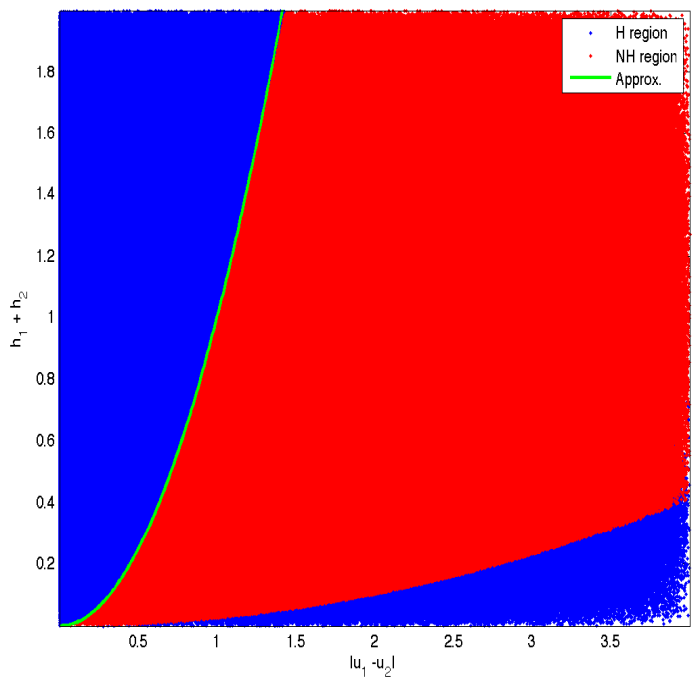

(b) Hyperbolicity region for $r=0.9$

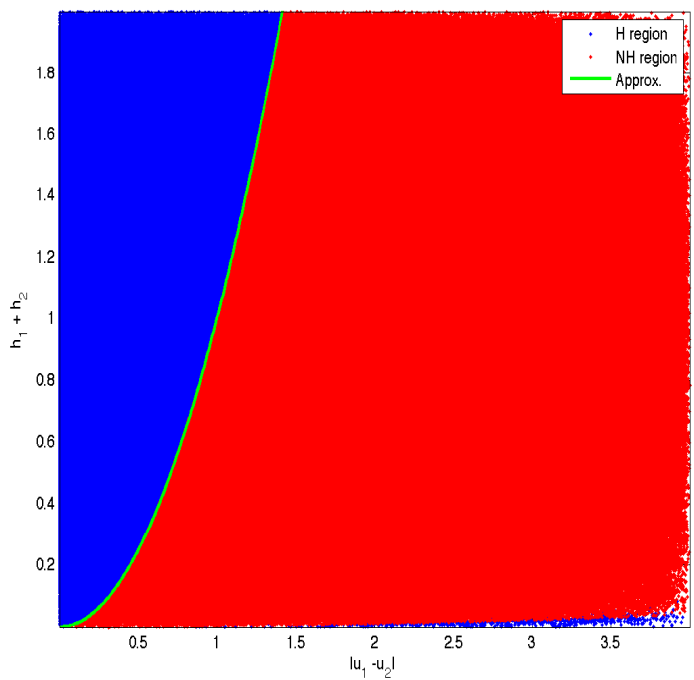

(d) Hyperbolicity region for $r=0.99$

Figure 2: Hyperbolicity regions of the two-layer shallow-water system in the plane $\left(\left|\hat{u}_{1}-\hat{u}_{2}\right|, \hat{h}_{1}+\hat{h}_{2}\right)$ : in blue the hyperbolic region, in red the non-hyperbolic regions and the green line is given by (2.8) 
one to define the nonconservative product $A(w) \cdot w_{x}$ as a bounded measure provided a family of Lipschitz continuous paths $\Phi:[0,1] \times \Omega \times \Omega \rightarrow \Omega$ is prescribed, which must satisfy certain natural regularity conditions, in particular

$$
\Phi\left(0 ; w_{L}, w_{R}\right)=w_{L}, \quad \Phi\left(1 ; w_{L}, w_{R}\right)=w_{R}
$$

and

$$
\Phi(s ; w, w)=w .
$$

Here, the family of straight segments is considered:

$$
\Phi\left(s ; w_{L}, w_{R}\right)=w_{L}+s\left(w_{R}-w_{L}\right) .
$$

We consider here some path-conservative numerical schemes in the sense defined in [11], that is, numerical schemes of the general form:

$$
w_{i}^{n+1}=w_{i}^{n}-\frac{\Delta t}{\Delta x}\left(D_{i-1 / 2}^{+}+D_{i+1 / 2}^{-}\right),
$$

where $\Delta x$ and $\Delta t$ are, for simplicity, assumed to be constant; $w_{i}^{n}$ is the approximation provided by the numerical scheme of the cell average of the exact solution at the $i$-th cell, $I_{i}=\left[x_{i-1 / 2}, x_{i+1 / 2}\right]$ at the $n$-th time level $t^{n}=n \Delta t$, and

$$
D_{i+1 / 2}^{ \pm}=D^{ \pm}\left(w_{i}^{n}, w_{i+1}^{n}\right)
$$

where $D^{-}$and $D^{+}$are two Lipschitz continuous functions from $\Omega \times \Omega$ to $\Omega$ satisfying:

$$
D^{ \pm}(w, w)=0, \quad \forall w \in \Omega
$$

and for every $w_{L}, w_{R} \in \Omega$,

$$
D^{-}\left(w_{L}, w_{R}\right)+D^{+}\left(w_{L}, w_{R}\right)=\int_{0}^{1} \mathcal{A}\left(\Phi\left(s ; w_{L}, w_{R}\right)\right) \frac{\partial \Phi}{\partial s}\left(s ; w_{L}, w_{R}\right) d s .
$$

These conditions provide a generalization of the concept of conservative scheme introduced by Lax for systems of conservation laws. In particular, if the system (2.2) admits a conservative subsystem, a path-conservative numerical scheme is conservative in the sense of Lax for that subsystem. The influence of the family of paths in the numerical approximation of shocks and the difficulties related to the convergence to the weak solutions of the system have been discussed in [5].

The family of generalized Roe schemes introduced in [14] constitutes a particular case of path-conservative numerical methods. These schemes are based on the general concept of Roe linearization for (2.2) introduced in [14]: given a family of paths $\Phi$, a function $A_{\Phi}: \Omega \times \Omega \mapsto \mathcal{M}_{4 \times 4}(\mathbb{R})$ is called a Roe linearization if it verifies the following properties:

- for any $w_{L}, w_{R} \in \Omega, A_{\Phi}\left(w_{L}, w_{R}\right)$ has 4 distinct real eigenvalues, 
- for every $w \in \Omega$,

$$
A_{\Phi}(w, w)=A(w)
$$

- for any $w_{L}, w_{R} \in \Omega$,

$$
A_{\Phi}\left(w_{L}, w_{R}\right) \cdot\left(w_{R}-w_{L}\right)=\int_{0}^{1} \mathcal{A}\left(\Phi\left(s ; w_{L}, w_{R}\right)\right) \frac{\partial \Phi}{\partial s}\left(s ; w_{L}, w_{R}\right) d s .
$$

The Roe linearization considered here is the following (see [12]): given two states

$$
w_{L}=\left[\begin{array}{c}
h_{1}^{L} \\
q_{1}^{L} \\
h_{2}^{L} \\
q_{2}^{L}
\end{array}\right], \quad w_{R}=\left[\begin{array}{c}
h_{1}^{R} \\
q_{1}^{R} \\
h_{2}^{R} \\
q_{2}^{R}
\end{array}\right]
$$

the matrix $A_{\Phi}\left(w_{L}, w_{R}\right)$ is defined by:

$$
\left[\begin{array}{cccc}
0 & 1 & 0 & 0 \\
-\left(\hat{u}_{1}\right)^{2}+\left(\hat{c}_{1}\right)^{2} & 2 \hat{u}_{1} & \left(\hat{c}_{1}\right)^{2} & 0 \\
0 & 0 & 0 & 1 \\
r\left(\hat{c}_{2}\right)^{2} & 0 & -\left(\hat{u}_{2}\right)^{2}+\left(\hat{c}_{2}\right)^{2} & 2 \hat{u}_{2}
\end{array}\right]
$$

where

$$
\hat{u}_{k}=\frac{\sqrt{h_{k}^{L}} u_{k}^{L}+\sqrt{h_{k}^{R}} u_{k}^{R}}{\sqrt{h_{k}^{L}}+\sqrt{h_{k}^{R}}}, \quad \hat{c}_{k}=\sqrt{g \frac{h_{k}^{L}+h_{k}^{R}}{2}}, \quad k=1,2
$$

with

$$
u_{k}^{L}=\frac{q_{k}^{L}}{h_{k}^{L}}, \quad u_{k}^{R}=\frac{q_{k}^{R}}{h_{k}^{R}}, \quad k=1,2 .
$$

Once the Roe linearization has been chosen, the numerical scheme is given by (3.3) with:

$$
D_{i+1 / 2}^{ \pm}=A_{\Phi}^{ \pm}\left(w_{i}^{n}, w_{i+1}^{n}\right) \cdot\left(w_{i+1}^{n}-w_{i}^{n}\right)
$$

where

$$
A_{\Phi}^{ \pm}\left(w_{L}, w_{R}\right)=\frac{1}{2}\left(A_{\Phi}\left(w_{L}, w_{R}\right) \pm\left|A_{\Phi}\left(w_{L}, w_{R}\right)\right|\right) .
$$

The absolute value of the intermediate matrix is given by:

$$
\left|A_{\Phi}\left(w_{L}, w_{R}\right)\right|=K_{\Phi}\left(w_{L}, w_{R}\right) \cdot\left|L_{\Phi}\left(w_{L}, w_{R}\right)\right| \cdot\left(K_{\Phi}\left(w_{L}, w_{R}\right)\right)^{-1}
$$

with

$$
\left|L_{\Phi}\left(w_{L}, w_{R}\right)\right|=\left[\begin{array}{ccc}
\left|\lambda_{\Phi}^{1}\left(w_{L}, w_{R}\right)\right| & & 0 \\
0 & \ddots & \\
& & \left|\lambda_{\Phi}^{4}\left(w_{L}, w_{R}\right)\right|
\end{array}\right]
$$

being $\lambda_{\Phi}^{1}\left(w_{L}, w_{R}\right), \cdots, \lambda_{\Phi}^{4}\left(w_{L}, w_{R}\right)$ the eigenvalues of $A_{\Phi}\left(w_{L}, w_{R}\right)$ and $K_{\Phi}\left(w_{L}, w_{R}\right)$ a $4 \times 4$ matrix whose columns are associated eigenvectors. 
When $A_{\Phi}\left(w_{L}, w_{R}\right)$ has complex eigenvalues, this matrix is no more a Roe linearization in the sense of the definition above, as the first requirement is not satisfied. Nevertheless, the numerical scheme (3.3),(3.8),(3.9) can still be applied by redefining the meaning of the absolute value of the intermediate matrix. To do this, we use the real Jordan decomposition of $A_{\Phi}\left(w_{L}, w_{R}\right)$, that is,

$$
A_{\Phi}\left(w_{L}, w_{R}\right)=K_{\Phi}^{J}\left(w_{L}, w_{R}\right) \cdot L_{\Phi}^{J}\left(w_{L}, w_{R}\right) \cdot\left(K_{\Phi}^{J}\left(w_{L}, w_{R}\right)\right)^{-1}
$$

where $L_{\Phi}^{J}\left(w_{L}, w_{R}\right)$ is a block diagonal matrix whose diagonal blocks are either the real eigenvalues or $2 \times 2$ blocks of the form:

$$
\left[\begin{array}{cc}
\alpha & \beta \\
-\beta & \alpha
\end{array}\right]
$$

associated to every pair of conjugate complex eigenvalues $\alpha \pm \mathrm{i} \beta . K_{\Phi}^{J}\left(w_{L}, w_{R}\right)$ is the real matrix corresponding to the change of basis. Now, $\left|A_{\Phi}\left(w_{L}, w_{R}\right)\right|$ can be formally defined by replacing in $(3.10) K_{\Phi}\left(w_{L}, w_{R}\right)$ by $K_{\Phi}^{J}\left(w_{L}, w_{R}\right)$ and $\left|L_{\Phi}\left(w_{L}, w_{R}\right)\right|$ by the diagonal matrix obtained from the Jordan matrix by taking the absolute values of the real eigenvalues and by replacing the diagonal blocks (3.11) corresponding to a pair of conjugate complex eigenvalues by the diagonal block:

$$
\left[\begin{array}{cc}
\sqrt{\alpha^{2}+\beta^{2}} & 0 \\
0 & \sqrt{\alpha^{2}+\beta^{2}}
\end{array}\right] .
$$

The technique introduce here to enforce the hyperbolic character can be also applied to the more family of path-conservative numerical methods introduced in [7] corresponding to the choice:

$$
D_{i+1 / 2}^{ \pm}=\widehat{A}_{\Phi}^{ \pm}\left(w_{i}^{n}, w_{i+1}^{n}\right) \cdot\left(w_{i+1}^{n}-w_{i}^{n}\right),
$$

where

$$
A_{\Phi}\left(W_{L}, W_{R}\right)=\widehat{A}_{\Phi}^{+}\left(w_{L}, w_{R}\right)+\widehat{A}_{\Phi}^{-}\left(w_{L}, w_{R}\right)
$$

is any decomposition of the Roe linearization of the form:

$$
\widehat{A}_{\Phi}^{ \pm}\left(w_{L}, w_{R}\right)=\frac{1}{2}\left(A_{\Phi}\left(w_{L}, w_{R}\right) \pm Q_{\Phi}\left(w_{L}, w_{R}\right)\right)
$$

where $Q_{\Phi}\left(w_{L}, w_{R}\right)$ can be interpreted as the numerical viscosity matrix. Different numerical schemes can be obtained by considering different viscosity matrices $Q_{\Phi}\left(w_{L}, w_{R}\right)$ :

- Roe scheme corresponds to the choice

$$
Q_{\Phi}\left(w_{L}, w_{R}\right)=\left|A_{\Phi}\left(w_{L}, w_{R}\right)\right| .
$$


- Lax-Friedrichs scheme corresponds to the choice

$$
Q_{\Phi}\left(w_{L}, w_{R}\right)=\frac{\Delta x}{\Delta t} I d
$$

being $I d$ the identity matrix.

- An extension of Lax-Wendroff scheme to non-conservative systems corresponds the choice

$$
Q_{\Phi}\left(w_{L}, w_{R}\right)=\frac{\Delta t}{\Delta x} A_{\Phi}^{2}\left(w_{L}, w_{R}\right) .
$$

- FORCE and GFORCE schemes correspond to the choice

$$
Q_{\Phi}\left(w_{L}, w_{R}\right)=(1-\omega) \frac{\Delta x}{\Delta t} I d+\omega \frac{\Delta t}{\Delta x} A_{\Phi}^{2}\left(w_{L}, w_{R}\right),
$$

with $\omega=0.5$ and $\omega=\frac{1}{1+C F L}$ respectively.

These generalized Lax-Friedirchs, FORCE, or GFORCE schemes are more diffusive than Roe scheme but they are less costly, as the calculation of the eigenstructure of the intermediate matrices is not required for their implementation. This is specially advantageous when they are extended to high order by means of a reconstruction operator, as the results provided by these schemes are very similar to those obtained with the high order extension of Roe, being the computational cost significantly lower: see [7] for detailss.

Notice that Lax-Friedrichs scheme can be applied without any modification when $A_{\Phi}\left(w_{L}, w_{R}\right)$ has complex eigenvalues. In the case of Lax-Wendroff, FORCE, or GFORCE scheme, when complex eigenvalues appear we replace $A_{\Phi}^{2}\left(w_{L}, w_{R}\right)$ by:

$$
K_{\Phi}^{J}\left(w_{L}, w_{R}\right) \cdot\left(L_{\Phi}^{J}\left(w_{L}, w_{R}\right)\right)^{2} \cdot\left(K_{\Phi}^{J}\left(w_{L}, w_{R}\right)\right)^{-1} .
$$

When these modifications are done, all of these numerical schemes can be still applied when complex eigenvalues are present. Nevertheless, they are not in general $\mathrm{L}^{2}$ linearly stable. In effect, let us consider a linear system

$$
w_{t}+A \cdot w_{x}=0,
$$

where $A$ has complex eigenvalues. By simplicity, let us suppose that $A \in \mathcal{M}_{4 \times 4}(\mathbb{R})$ having two real eigenvalues and two complex ones: $\lambda_{1}, \lambda_{2} \in \mathbb{R}$ and

$$
\lambda_{3}=\alpha+\mathrm{i} \beta, \quad \lambda_{4}=\alpha-\mathrm{i} \beta, \quad \alpha, \beta \in \mathbb{R}
$$

Let us consider $A=K \cdot L^{J} \cdot K^{-1}$, being

$$
L^{J}=\left(\begin{array}{cccc}
\lambda_{1} & 0 & 0 & 0 \\
0 & \lambda_{2} & 0 & 0 \\
0 & 0 & \alpha & \beta \\
0 & 0 & -\beta & \alpha
\end{array}\right)
$$


Observe that the numerical scheme (3.3)-(3.12)-(3.14) can be written as

$$
w_{i}^{n+1}=\frac{\Delta t}{2 \Delta x}(A+Q) w_{i-1}^{n}+\left(1-\frac{\Delta t}{\Delta x} Q\right) w_{i}^{n}-\frac{\Delta t}{2 \Delta x}(A-Q) w_{i+1}^{n} .
$$

The corresponding amplification matrix is given by,

$$
G^{a}(\xi)=\frac{\Delta t}{2 \Delta x}(A+Q) \mathrm{e}^{-\mathrm{i} \xi \Delta x}+\left(1-\frac{\Delta t}{\Delta x} Q\right)-\frac{\Delta t}{2 \Delta x}(A-Q) \mathrm{e}^{\mathrm{i} \xi \Delta x} .
$$

Let us consider a viscosity matrix of the form:

$$
Q=K \cdot L_{Q} \cdot K^{-1}
$$

where

$$
L_{Q}=\left(\begin{array}{cccc}
d_{1} & 0 & 0 & 0 \\
0 & d_{2} & 0 & 0 \\
0 & 0 & \chi & \delta \\
0 & 0 & -\delta & \chi
\end{array}\right), \quad d_{1}, d_{2}, \chi, \delta \in \mathbb{R}
$$

The following result can be obtained:

THEOREM 1. Let us consider $Q$ given by (3.20)-(3.21). If

$$
\left(\frac{\Delta t}{\Delta x} \lambda_{j}\right)^{2} \leq \frac{\Delta t}{\Delta x} d_{j} \leq 1 \quad j=1,2
$$

and

$$
\frac{\Delta t}{\Delta x}\left(\alpha^{2}+\beta^{2}\right) \leq \chi, \quad \frac{\Delta t}{\Delta x}\left(\chi^{2}+\delta^{2}\right) \leq \chi,
$$

then the following inequality holds,

$$
\rho\left(G^{a}(\xi)\right)^{2} \leq 1+\varepsilon_{o}, \quad \text { with } \quad \varepsilon_{o}=2 \frac{\Delta t}{\Delta x} \max \left(2|\delta|, \frac{\beta^{2}}{\sqrt{\beta^{2}-\delta^{2}}}\right) .
$$

From the point of view of the $L^{2}$ stability the optimal choice of $\delta$ is $\delta=0$. In this case the inequalities (3.23) reduce to

$$
\left(\frac{\Delta t}{\Delta x}\right)^{2}\left(\alpha^{2}+\beta^{2}\right) \leq \frac{\Delta t}{\Delta x} \chi \leq 1
$$

But even if $\delta=0$ and $\chi$ satisfies (3.24), the $\mathrm{L}^{2}$ stability is not obtained, as we have:

$$
\rho\left(G^{a}(\xi)\right)^{2} \leq 1+2 \frac{\Delta t}{\Delta x}|\beta| .
$$

We list hereafter the choices of $d_{1}, d_{2}$, and $\chi$ corresponding to the numerical schemes described above for the nonlinear system. In any case the inequalities (3.22) and (3.24) are satisfied provided that the following CFL condition is prescribed:

$$
\frac{\Delta t}{\Delta x} \max \left(\left|\lambda_{1}\right|,\left|\lambda_{2}\right|, \sqrt{\alpha^{2}+\beta^{2}}\right) \leq 1
$$


- Roe scheme (3.15):

$$
d_{j}=\left|\lambda_{j}\right|, j=1,2, \chi=\sqrt{\alpha^{2}+\beta^{2}} .
$$

- Lax-Friedrich scheme (3.16):

$$
d_{1}=d_{2}=\chi=\frac{\Delta x}{\Delta t}
$$

- Lax-Wendroff scheme (3.17):

$$
d_{j}=\frac{\Delta t}{\Delta x} \lambda_{j}^{2}, j=1,2, \chi=\frac{\Delta t}{\Delta x}\left(\alpha^{2}+\beta^{2}\right) .
$$

- FORCE or (GFORCE) schemes (3.18):

$$
d_{j}=(1-\omega) \frac{\Delta x}{\Delta t}+\omega \frac{\Delta t}{\Delta x} \lambda_{j}^{2}, j=1,2, \chi=(1-\omega) \frac{\Delta x}{\Delta t}+\omega \frac{\Delta t}{\Delta x}\left(\alpha^{2}+\beta^{2}\right) .
$$

In general, the bound $\rho\left(G^{a}(\xi)\right)^{2} \leq 1+2 \frac{\Delta t}{\Delta x}|\beta|$ cannot be expected to be improved. To see this, let us consider the simpler system with complex eigenvalues,

$$
w_{t}+A \cdot w_{x}=0,
$$

with $A \in \mathcal{M}_{2 \times 2}(\mathbb{R})$ is given by:

$$
A=\left(\begin{array}{cc}
\alpha & \beta \\
-\beta & \alpha
\end{array}\right), \quad \alpha, \beta \in \mathbb{R} .
$$

If $A$ is decomposed as $A=K \cdot L \cdot K^{-1}$ with $\mathrm{E}=\operatorname{diag}\left(\lambda_{1}, \lambda_{2}\right), \lambda_{1,2}=\alpha \pm \mathrm{i} \beta$ and

$$
K=\left(\begin{array}{cc}
1 & 1 \\
\mathrm{i} & -\mathrm{i}
\end{array}\right)
$$

and the change of variables $\widetilde{w}=K^{-1} w$ is used, the system reduces to:

$$
\frac{\partial \widetilde{w}_{k}}{\partial t}+\lambda_{k} \frac{\partial \widetilde{w}_{k}}{\partial x}=0, \quad k=1,2,
$$

whose solution is:

$$
\widetilde{w}_{1}=C_{1} \mathrm{e}^{\mathrm{i} \sigma\left(x-\lambda_{1} t\right)}=C_{1} \mathrm{e}^{\sigma \beta t} \mathrm{e}^{\mathrm{i} \sigma(x-\alpha t)}, \quad k=1,2,
$$

where $C_{1}$ is an arbitrary complex number. The solution for $\widetilde{w}_{2}$ is similar. Notice that the solution (3.27) increase exponentially in time depending on $\beta$, so that is expectable to find a similar behavior in the numerical solutions. Therefore, in order to obtain the $L^{2}$ stability it is necessary to modify the system to get rid of this exponential growing.

Remark 1. While these schemes are unstable for linear systems, in the nonlinear case they may remain stable in the presence of complex eigenvalues, as it will be seen in Section 5. Nevertheless, strong unphysical oscillations may appear in the numerical solutions when the modulus of the complex eigenvalues are big enough. 


\section{Numerical treatment of the loss of hyperbolic- ity}

In the previous Section we have presented some Roe-based numerical schemes that can be used even if the intermediate matrices have complex eigenvalues. In this section we describe a strategy to recover the hyperbolic character of the two-layer shallow-water system by a predictor/corrector strategy. Let us suppose that the approximations at time $t^{n}, w_{i}^{n}=\left[h_{1, i}^{n}, q_{1, i}^{n}, h_{2, i}^{n}, q_{2, i}^{n}\right]^{T}$, are known and let $\Delta t$ the time step. In order to compute $w_{i}^{n+1}$ we proceed as follows:

1. Predictor step: one of the numerical schemes introduced in the previous Section is applied to system (2.2) to obtain a first set of approximations at time $t^{n+1}, w_{i}^{*}=\left[h_{1, i}^{n+1}, q_{1, i}^{*}, h_{2, i}^{n+1}, q_{2, i}^{*}\right]^{T}$.

2. Corrector step: at every cell the hyperbolic character of the system is checked by verifying if the inequality $(1.4)$ is satisfied for the state $w_{i}^{*}$. To do this, we compute:

$$
C_{i}^{*}=\frac{\left(u_{1, i}^{*}-u_{2, i}^{*}\right)^{2}}{g^{\prime}\left(h_{1, i}^{n+1}+h_{2, i}^{n+1}\right)}-1,
$$

being $u_{k, i}^{*}=q_{k, i}^{*} / h_{k, i}^{n+1}$. Then the approximation at the $i$-th cell at time $t^{n+1}$ is calculated as follows:

- if $C_{i}^{*}<0$, then $w_{i}^{n+1}=w_{i}^{*}$,

- otherwise $w_{i}^{n+1}=\left[h_{1, i}^{n+1}, q_{1, i}^{n+1}, h_{2, i}^{n+1}, q_{2, i}^{n+1}\right]$, where

$$
q_{1, i}^{n+1}=h_{1, i}^{n+1} \cdot u_{i, 1}^{n+1}, \quad q_{2, i}^{n+1}=h_{2, i}^{n+1} \cdot u_{2, i}^{n+1},
$$

and $u_{j, i}^{n+1}, j=1,2$ are obtained by solving the system:

$$
\left\{\begin{array}{l}
u_{1, i}^{n+1}=u_{1, i}^{*}-\Delta t \frac{\bar{c}}{h_{1}^{n+1}}\left|u_{1, i}^{n}-u_{2, i}^{n}\right|\left(u_{1, i}^{n+1}-u_{2, i}^{n+1}\right) \\
u_{2, i}^{n+1}=u_{2, i}^{*}+r \Delta t \frac{\bar{c}}{h_{2}^{n+1}}\left|u_{1, i}^{n}-u_{2, i}^{n}\right|\left(u_{1, i}^{n+1}-u_{2, i}^{n+1}\right) \\
\frac{\left(u_{1, i}^{n+1}-u_{2, i}^{n+1}\right)^{2}}{g^{\prime}\left(h_{1, i}^{n+1}+h_{2, i}^{n+1}\right)}=1
\end{array}\right.
$$

for the unknowns $\bar{c}, u_{j, i}^{n+1}, j=1,2$.

Notice that the two first equations in this system correspond to adding a quadratic friction force between the layers in a split semi-implicit manner, and the third equation is used to calibrate the constant in the quadratic law so that the new cell averages are in the boundary of the hyperbolicity 
region, i.e. in the curve in which the system has a double and two simple real eigenvalues.

Some straightforward calculations allow us to obtain the value of $\bar{c}$ :

$$
\bar{c}=\frac{h_{1, i}^{n+1} h_{2, i}^{n+1}}{\Delta t\left(h_{2, i}^{n+1}+r h_{1, i}^{n+1}\right)\left|u_{1, i}^{n}-u_{2, i}^{n}\right|}\left(\frac{\left|u_{1, i}^{*}-u_{2, i}^{*}\right|}{\sqrt{g^{\prime}\left(h_{1, i}^{n+1}-h_{2, i}^{n+1}\right)}}-1\right),
$$

and then the values of $u_{j, i}^{n+1}, j=1,2$ are easily obtained.

Remark 2. As the inequality (1.4) is only an approximated criterium of hiperbolicity, in the practical implementation the corrector step is performed if $C_{i}^{*}<\varepsilon$, where $\varepsilon$ is a small parameter (usually $\varepsilon=O\left(10^{-5}\right)$ ).

\section{$5 \quad$ Numerical tests}

\section{$5.1 \quad$ Test 1}

This test is designed to assess the strategy defined in Section 4 to enforce the hyperbolicity of the two-layer shallow-water system. To do this, we apply the predictor/corrector strategy to the system with an initial condition which is far beyond the hiperbolicity region. We consider a flat channel whose axis is given by the interval $[-5,5]$.

The initial condition is

$$
\begin{aligned}
& h_{1}(x, 0)=\left\{\begin{array}{ll}
0.4 & \text { if } x>-0.5 \text { and } x<0.5 \\
0.5 & \text { otherwise }
\end{array} \quad h_{2}(x, 0)=1.0-h_{1}(x, 0),\right. \\
& u_{1}(x, 0)=0.2, \quad u_{2}(x, 0)=-0.3 .
\end{aligned}
$$

Free boundary conditions are imposed. The CFL parameter is set to 0.9 and $\Delta x=$ 0.01 and $r=0.99$. Note that

$$
\frac{\left(u_{1}-u_{2}\right)^{2}}{g^{\prime}\left(h_{1}+h_{2}\right)}=0.5^{2} / 0.0981
$$

at every point at time $t=0$.

Figure 3 shows the free surface and the interface evolution from $t=0 \mathrm{~s}$ to $t=1 \mathrm{~s}$ obtained with Roe scheme without (left) or with (right) the corrector step described in Section 4. As expected, the initial perturbation grows in time when Roe scheme is applied, while this is not the case when the corrector step is performed. Similar results can be observed for the velocities (see Figure 4). Figure 5 shows the evolution of $\left(u_{1}-u_{2}\right)^{2} /\left(g^{\prime}\left(h_{1}+h_{2}\right)\right)$ from $t=0 s$ to $t=1 s$. As expected, when the friction terms are considered, the inequality $\left(u_{1}-u_{2}\right)^{2} /\left(g^{\prime}\left(h_{1}+h_{2}\right)\right) \leq 1$ is always satisfied.

In this case, the strong oscillations obtained with the Roe scheme without the corrector step remain bounded. Nevertheless these oscillations are unphysical: in 
real flows, shear instabilities induce either mixture or the development of complex patterns at the interface but not internal waves of such an amplitude. Instead, when the corrector step is applied these phenomena are neglected.

\section{$5.2 \quad$ Test 2}

In this test the axis of the channel is the interval $[0,10]$. The bottom topography is given by the function

$$
H(x)=1.0-0.5 e^{-(x-5.0)^{2}}
$$

The initial condition is $q_{1}(x, 0)=q_{2}(x, 0)=0$,

$$
h_{1}(x, 0)= \begin{cases}0.5 & \text { if } x<5 \\ 0.0 & \text { otherwise }\end{cases}
$$

and

$$
h_{2}(x, 0)=H(x)-h_{1}(x, 0) .
$$

The initial condition represents an internal dam witch is suddenly removed. The layers are then allowed to evolve until a stationary state is reached. As boundary conditions, the relation $q_{1}(\cdot, t)=-q_{2}(\cdot, t)$ is imposed at both ends, and the free surface is fixed to $z=0$ at $x=10$, that is $h_{1}(10, t)+h_{2}(10, t)-H(x)=0$. In the simulations shown in Figures 6-8, CFL $=0.9, r=0.99$ and $\Delta x=1 / 35$. In the reached stationary solution, the upper layer fluid flows from left to right and the second one from left to right. The flow is subcritical if $x \geq 5$ and critical at $x=5$. At the left of $x=5$, the flow is first supercritical and then the subcritical regime is recovered by a stationary internal hydraulic jump (see Figure 6). Concerning the hyperbolicity region, the flow is always in the hyperbolic region, except in a small area located in the supercritical region. In this particular case, Roe scheme without the correction stage is able to reach the stationary state although complex eigenvalues appear. Figure 7 shows the values of $\left(u_{1}-u_{2}\right)^{2} /\left(g^{\prime}\left(h_{1}+h_{2}\right)\right)$ in the $x-t$ space when the Roe scheme without (left) or with (right) the corrector step described in Section 4 is used. As expected, when the friction terms are considered, the inequality $\left(u_{1}-u_{2}\right)^{2} /\left(g^{\prime}\left(h_{1}+h_{2}\right)\right) \leq 1$ is always satisfied, while this is not the case when the corrector step is not performed, being 1.2866 the maximal value of $\left(u_{1}-u_{2}\right)^{2} /\left(g^{\prime}\left(h_{1}+h_{2}\right)\right)$. Notice that the regions of hyperbolic regimes are almost identical.

We have also considered the system with a small friction force given by a quadratic law (1.2) with $c_{0}=0.001$. Figure 8 shows the values of $\left(u_{1}-u_{2}\right)^{2} /\left(g^{\prime}\left(h_{1}+h_{2}\right)\right)$ when the Roe scheme in combination with the friction law (1.2) with $c_{0}=0.001$ is used. Note that the flow is not always hyperbolic, but the values $\left(u_{1}-u_{2}\right)^{2} /\left(g^{\prime}\left(h_{1}+h_{2}\right)\right)$ are smaller, being its maximal value 1.2689. As in the previous case, the regions of hyperbolic regime are practically the same in all the cases. 


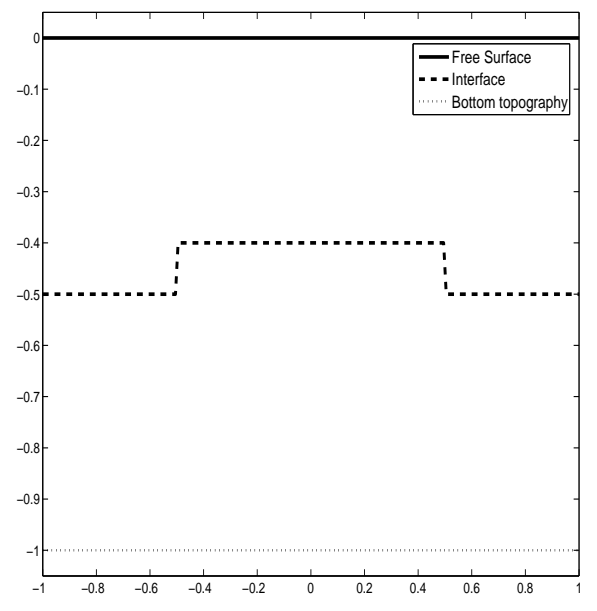

(a) $t=0 \mathrm{~s}$

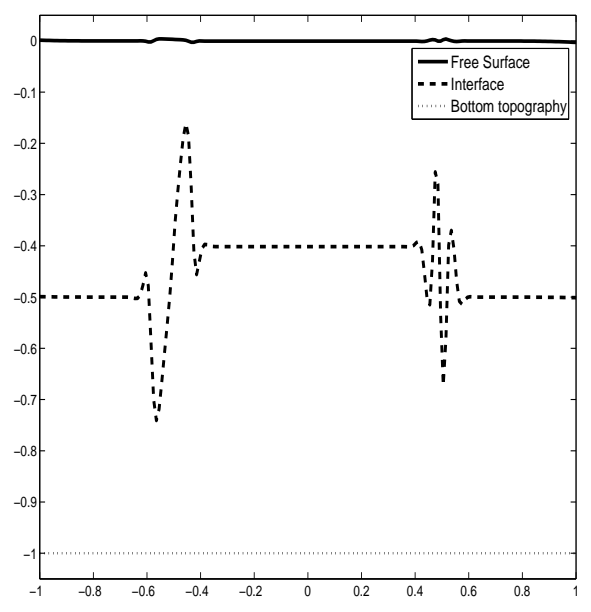

(c) $t=0.5 \mathrm{~s}$

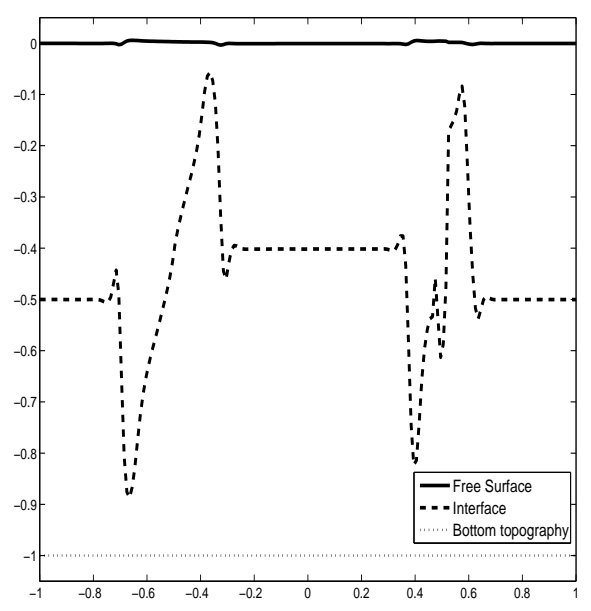

(e) $t=1.0 \mathrm{~s}$

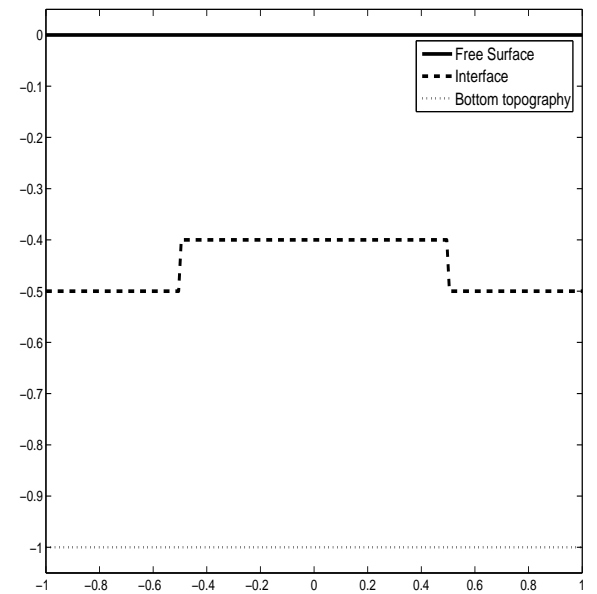

(b) $t=0 \mathrm{~s}$

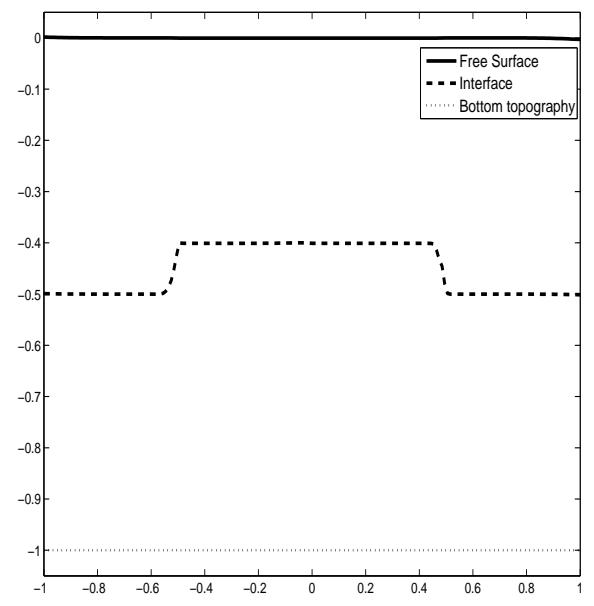

(d) $t=0.5 \mathrm{~s}$

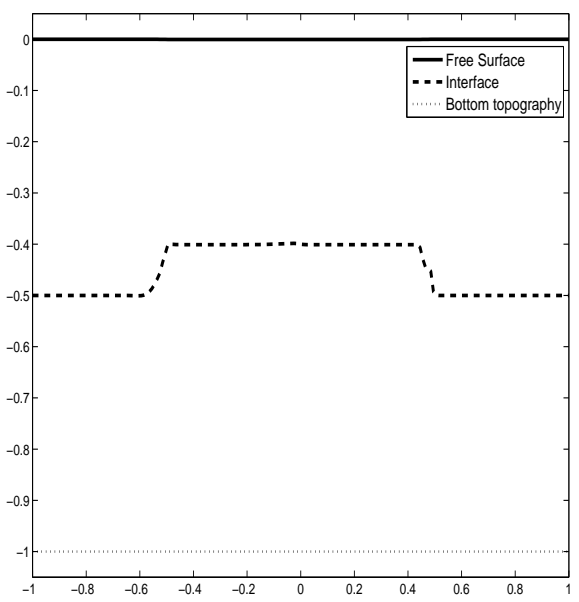

17

Figure 3: Test 1: Free surface and interface evolution. Roe scheme (left column). Roe scheme with friction to enforce the hyperbolicity region (right column) 


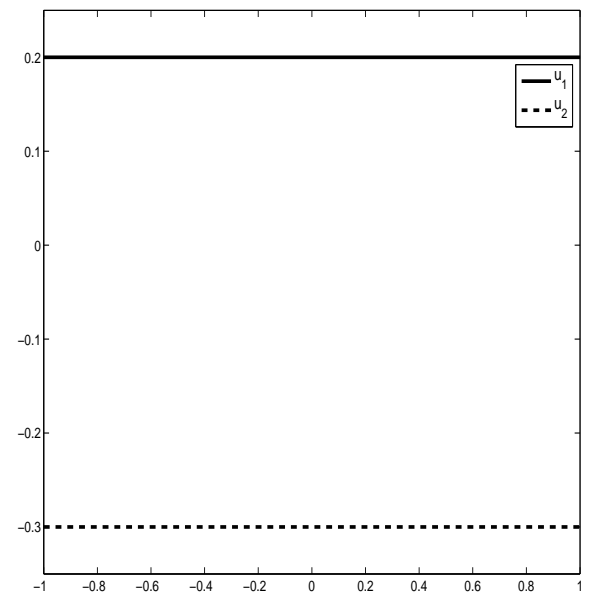

(a) $t=0 \mathrm{~s}$

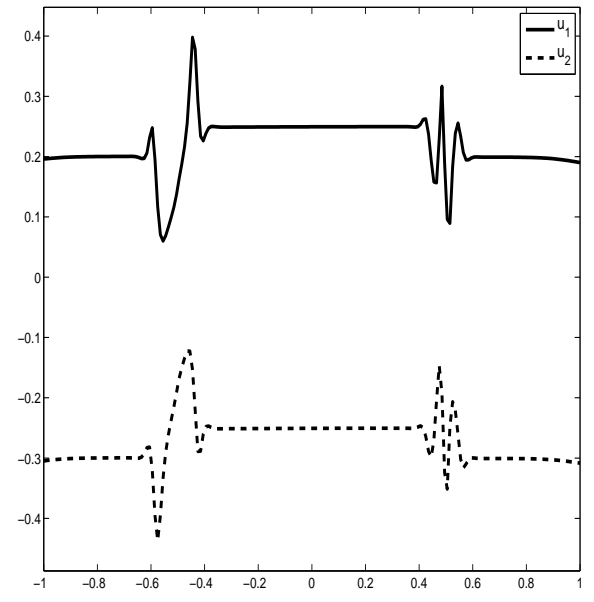

(c) $t=0.5 \mathrm{~s}$

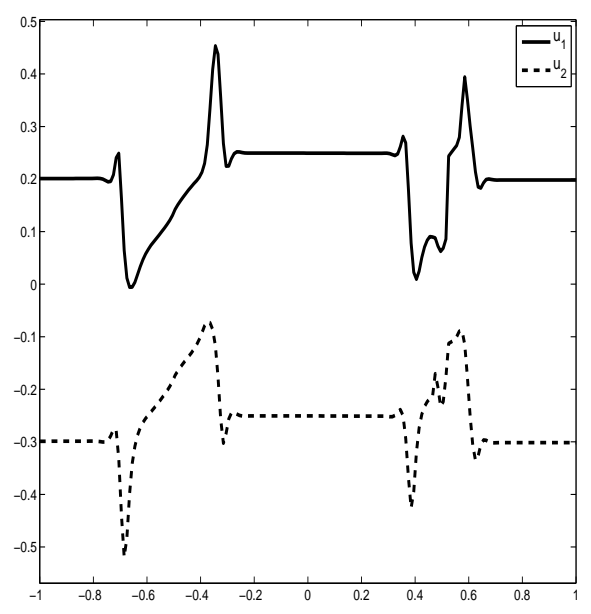

(e) $t=1.0 \mathrm{~s}$

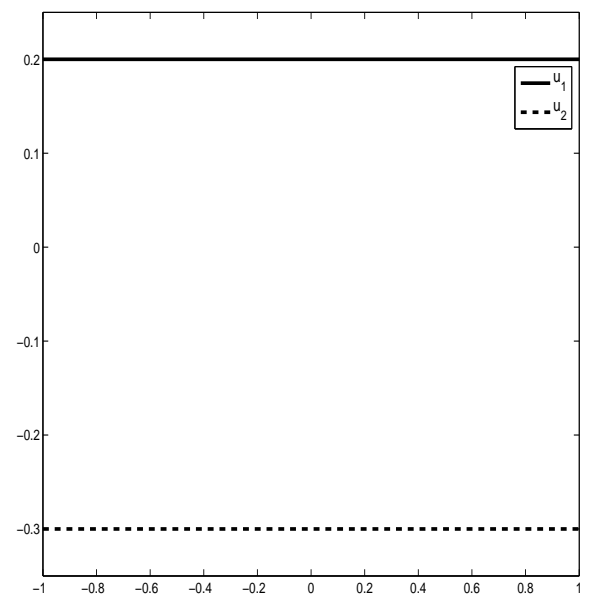

(b) $t=0 \mathrm{~s}$

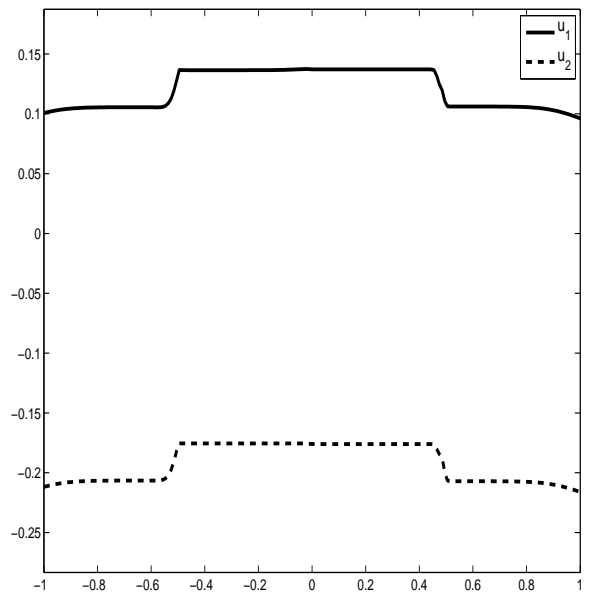

(d) $t=0.5 \mathrm{~s}$

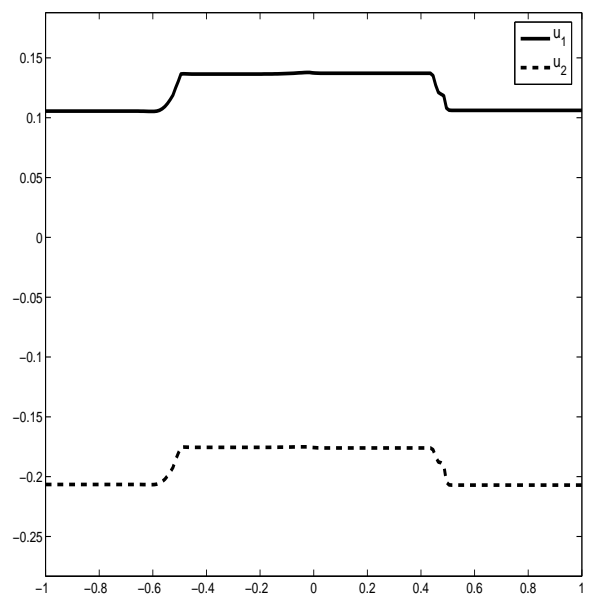

(f) $t=1.0 \mathrm{~s}$

Figure 4: Test 1: $u_{1}$ and $u_{2}$ evolution. Roe scheme (left column). Roe scheme with friction to enforce the hyperbolicity region (right column) 


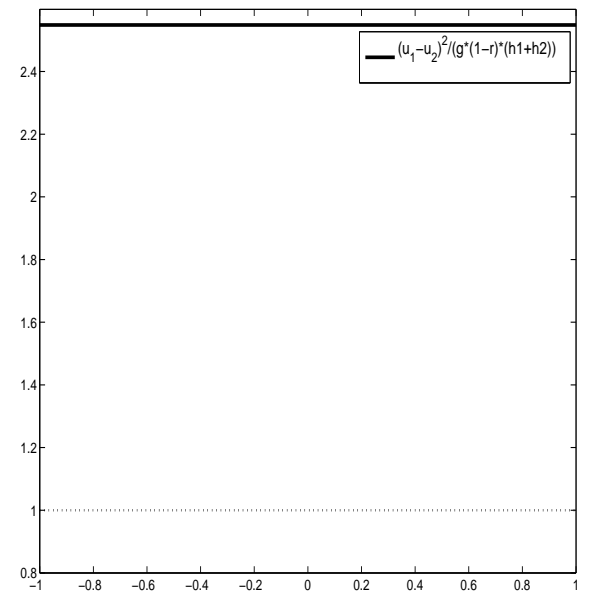

(a) $t=0 \mathrm{~s}$

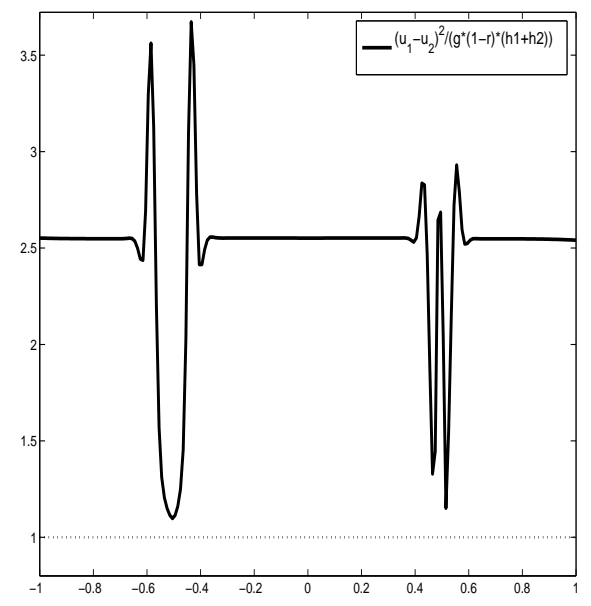

(c) $t=0.5 \mathrm{~s}$

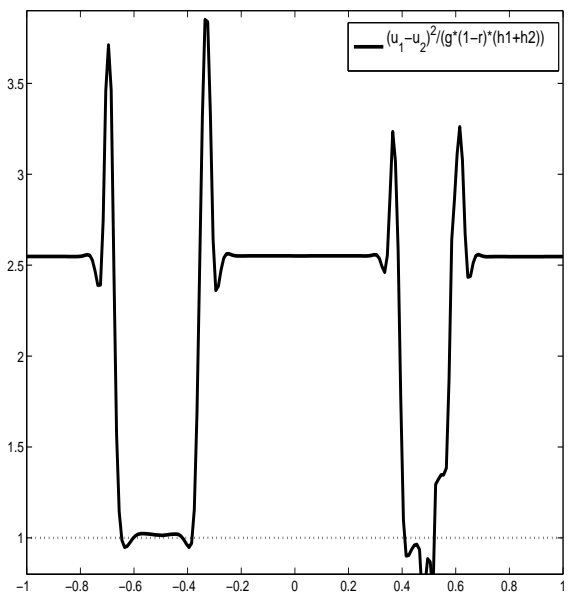

(e) $t=1.0 \mathrm{~s}$

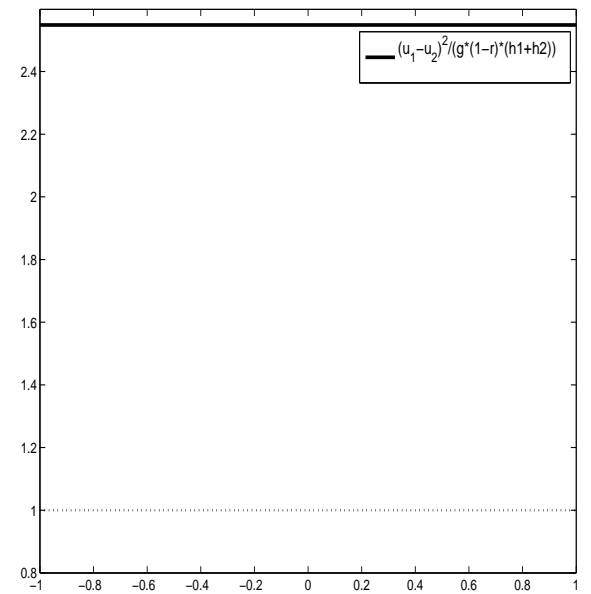

(b) $t=0 \mathrm{~s}$

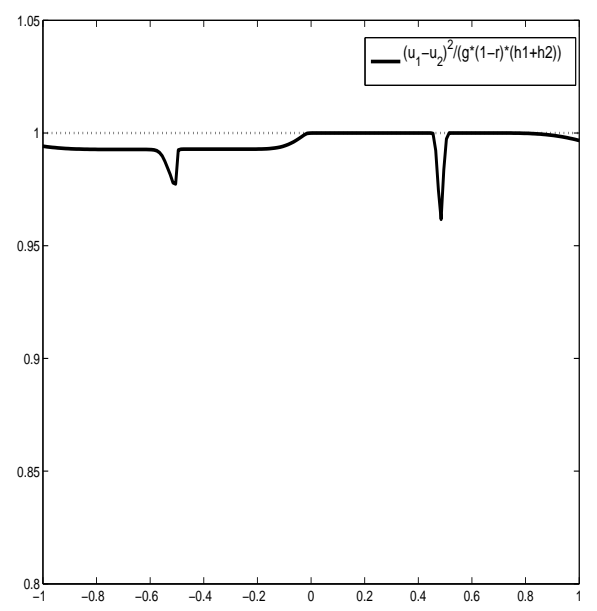

(d) $t=0.5 \mathrm{~s}$

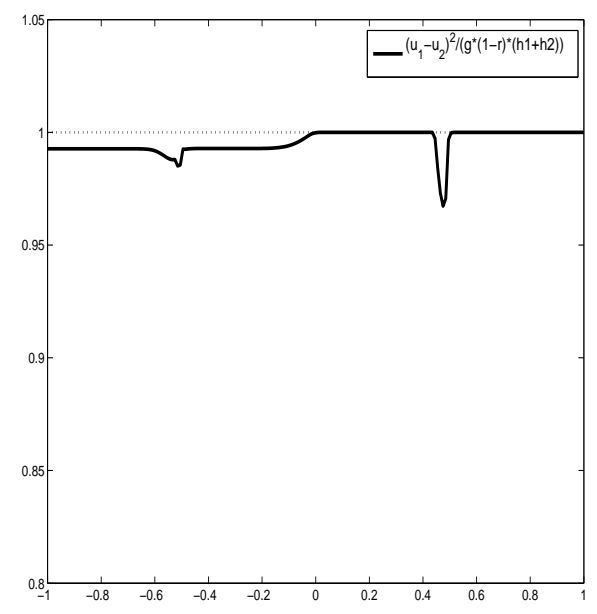

(f) $t=1.0 \mathrm{~s}$

Figure 5: Test 1: $\left(u_{1}-u_{2}\right) /\left(g^{\prime}\left(h_{1}+h_{2}\right)\right)$ evolution. Roe scheme (left column). Roe scheme with correction step to enforce the hyperbolicity region (right column) 


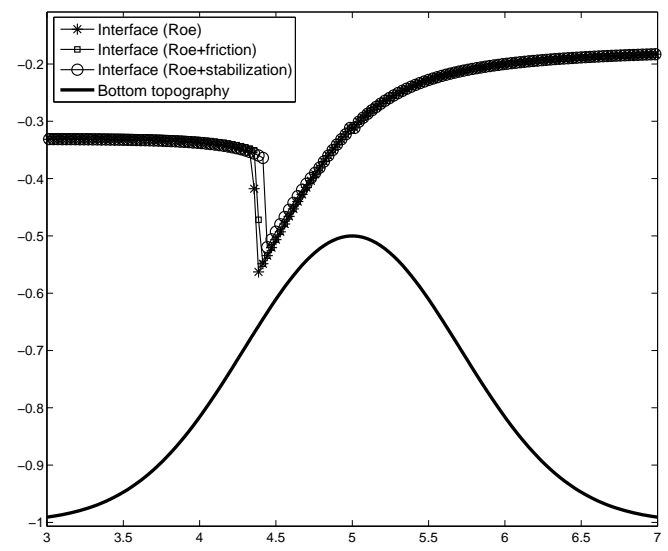

Figure 6: Test 2: Computed interface at the steady state: Roe scheme (line with stars), Roe scheme with internal friction (1.2) (line with squares), and Roe scheme with correction step (line with circles).

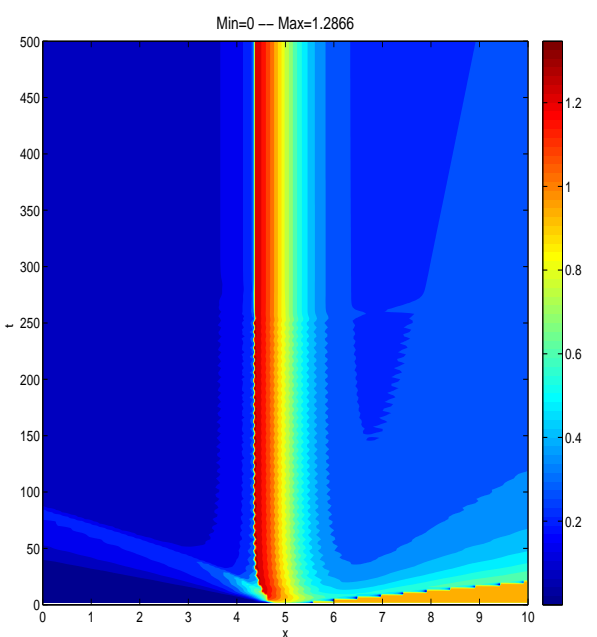

(a) $\left(u_{1}-u_{2}\right)^{2} /\left(g^{\prime}\left(h_{1}+h_{2}\right)\right)$. Roe scheme.

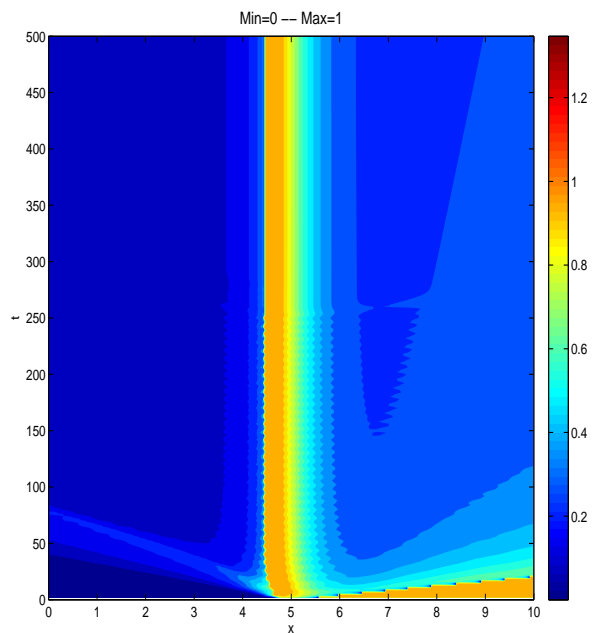

(b) $\left(u_{1}-u_{2}\right)^{2} /\left(g^{\prime}\left(h_{1}+h_{2}\right)\right)$. Roe scheme with correction step.

Figure 7: Test 2: $\left(u_{1}-u_{2}\right)^{2} /\left(g^{\prime}\left(h_{1}+h_{2}\right)\right)$ evolution. Roe scheme (left column). Roe scheme with correction step to enforce the hyperbolicity region (right column) 


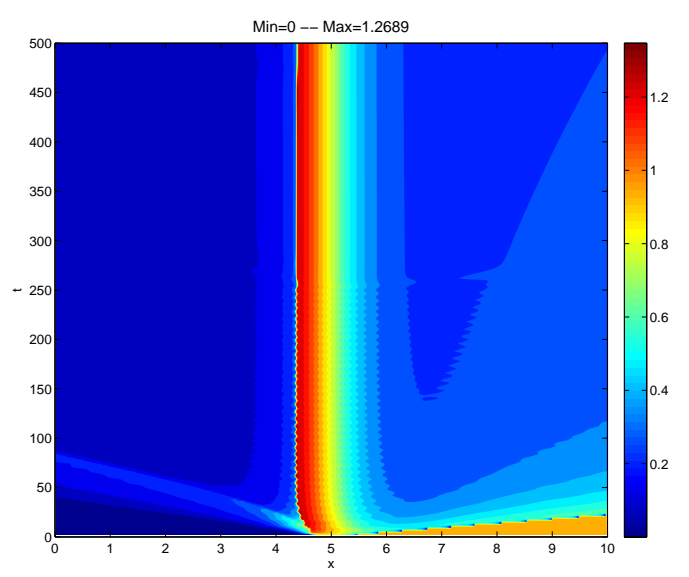

Figure 8: Test 2: $\left(u_{1}-u_{2}\right)^{2} /\left(g^{\prime}\left(h_{1}+h_{2}\right)\right)$ evolution. Roe scheme with internal friction (1.2) with $c_{0}=0.001$.

Figure 6 shows the comparison of the three computed interfaces at the steady state. Note that the only differences are the position and amplitude of the internal bore.

\section{$5.3 \quad$ Test 3}

This test is designed to assess the strategy defined in Section 4 to enforce the hyperbolicity region of the two-layer shallow-water system in the case of a barotropic tidal forcing. Let us consider the same channel of Test 2 and, as initial condition, the stationary state computed in Test 2 is imposed. The total water height

$$
\left(h_{1}+h_{2}\right)(10, t)=h_{1}^{0}+h_{2}^{0}+0.005 \cos \left(t / 50+\frac{\pi}{4}\right),
$$

is imposed at $x=10$, being $h_{i}^{0}, i=1,2$ the water depth of both layers at the stationary state. Free boundary condition is imposed at $x=0$. Again, $\mathrm{CFL}=0.9$, $r=0.99$, and $\Delta x=1 / 35$. The numerical test is run from $t=0$ to $t=10000 \mathrm{~s}$. As a consequence of the tidal forcing, the internal bore moves from left to right according to the tidal currents.

Figure 9 shows the position of the computed interface at two different times of the tidal period. Notice that the main differences of the Roe scheme with or without corrector step is the location and amplitude of the internal bore. If, again, a small friction force give by $(1.2)$ with $c_{0}=0.003$ is added to the system and Roe scheme is applied, the differences are bigger: the internal bore location and amplitudes are different but also significant differences can be observed at the subcritical regions (see Figure 9).

Figure 10 shows the values $\left(u_{1}-u_{2}\right)^{2} /\left(g^{\prime}\left(h_{1}+h_{2}\right)\right)$ in the $x-t$ space for several tidal periods when the Roe scheme without (left) or with (right) corrector step 


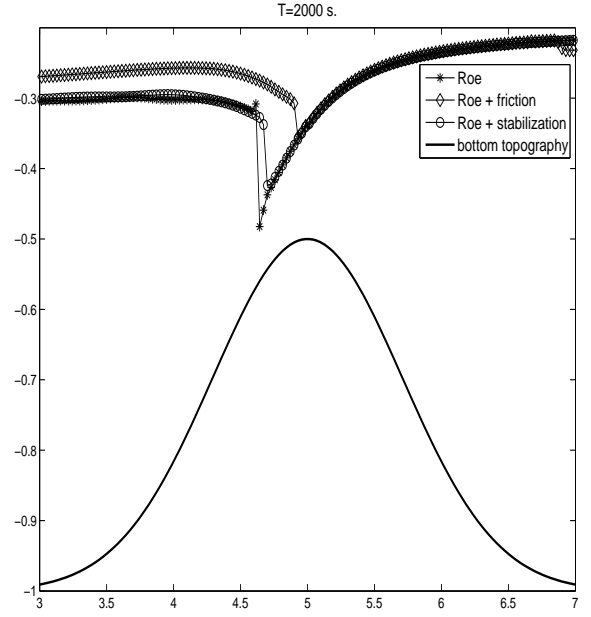

(a) $t=2000 \mathrm{~s}$.

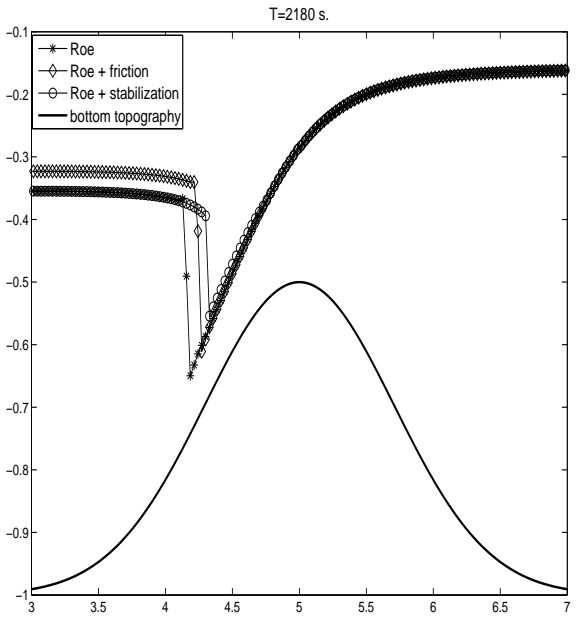

(b) $t=2180 \mathrm{~s}$.

Figure 9: Test 3: Computed interface at $t=2000 \mathrm{~s}$. (left) and $t=2180 \mathrm{~s}$. (right): Roe scheme (line with stars), Roe scheme with internal friction (1.2) (line with squares), and Roe scheme with correction step (line with circles).

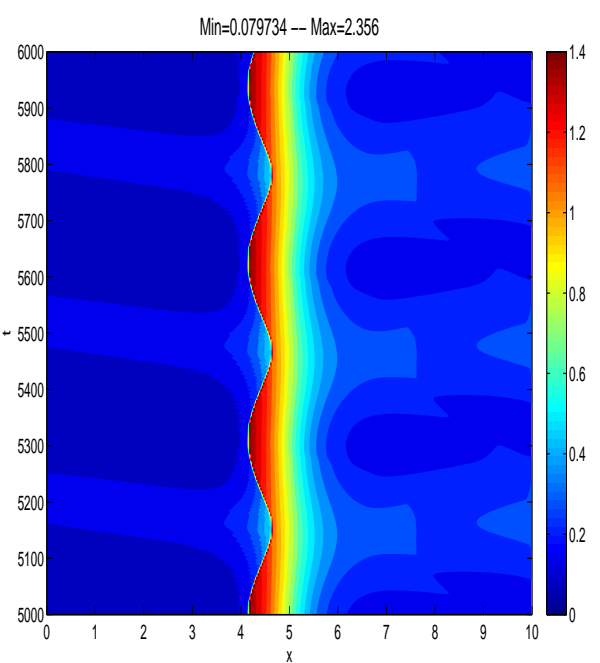

(a) $\left(u_{1}-u_{2}\right)^{2} /\left(g^{\prime}\left(h_{1}+h_{2}\right)\right)$. Roe scheme.

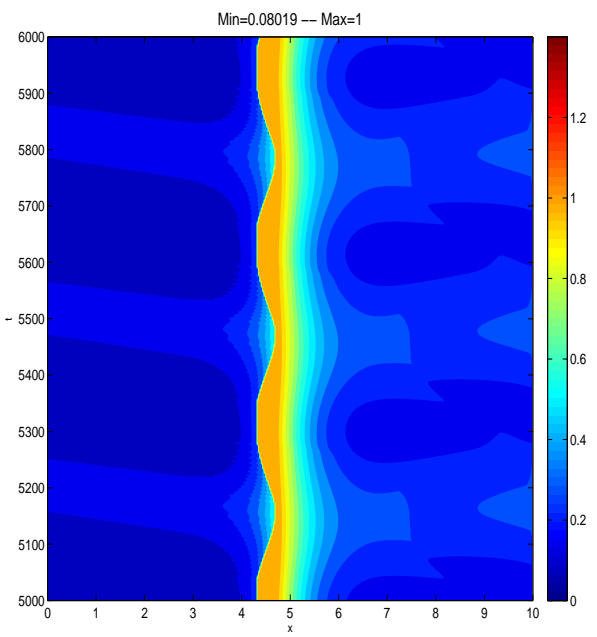

(b) $\left(u_{1}-u_{2}\right)^{2} /\left(g^{\prime}\left(h_{1}+h_{2}\right)\right)$. Roe scheme with correction step.

Figure 10: Test 3: $\left(u_{1}-u_{2}\right)^{2} /\left(g^{\prime}\left(h_{1}+h_{2}\right)\right)$ evolution. Roe scheme (left column). Roe scheme with correction step to enforce the hyperbolicity region (right column) 
described in Section 4 is used. As in the previous tests, when the corrector step is considered, the inequality $\left(u_{1}-u_{2}\right)^{2} /\left(g^{\prime}\left(h_{1}+h_{2}\right)\right) \leq 1$ is always satisfied, while is not the case when the Roe scheme is used without the corrector step. Note that in both Figures the regions of hyperbolic regime are almost identical.

Again, the simulation can be performed with Roe scheme without the corrector step. Nevertheless, some oscillations appear: a zoom of the region near the internal bore in Figure 9 can be seen in Figure 11(a). These oscillations increase as $r$ is closer to one: see Figure 11(b) corresponding to the same test problem with $r=0.999$

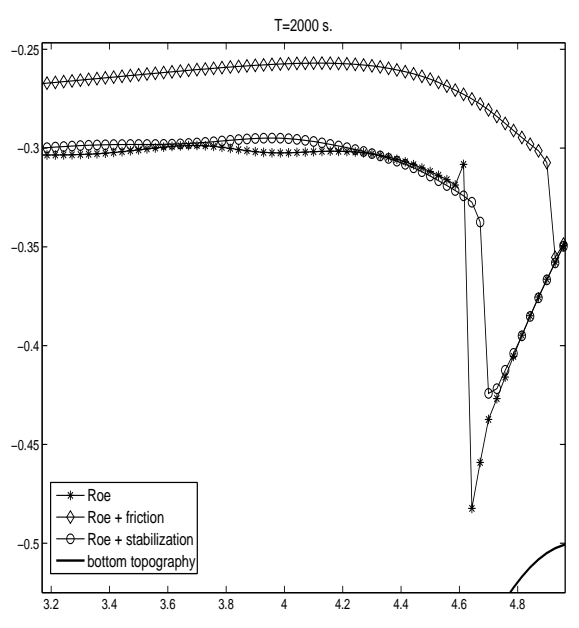

(a) $t=2000$ and $r=0.99$

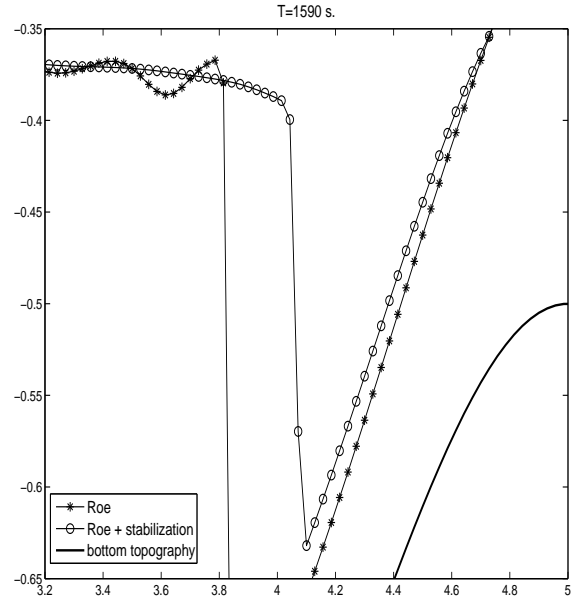

(b) $t=1590$ and $r=0.999$

Figure 11: Test 3:Computed interface at $t=2000 \mathrm{~s}$. with $r=0.99$ (left) and $t=1590 \mathrm{~s}$. (right) with $r=0.999$ : Roe scheme (line with stars) and Roe scheme with correction step (line with circles).

\section{Conclusions}

In this work, a characterization of the hyperbolicity region for the two layer shallowwater system is proposed and checked by using the first order approximation of the eigenvalues of the system given in [13] when $r \cong 1$.

Some path-conservative finite volume schemes in the sense defined in [11] have also been presented, that can be used even if the system is not hyperbolic, but they are not in general $\mathrm{L}^{2}$ stable.

The main contribution of this work is a simple but efficient strategy to enforce the hyperbolicity of the two-layer shallow-water system consisting in adding to the system an extra amount of friction at every cell in which complex eigenvalues are detected at a given time step. The implementation is performed by a predictor/corrector strategy: first a numerical scheme is applied to the unmodified 
two-layer system, regardless of the hyperbolic character of the system. Next, we check if the predicted cell averages are in the hyperbolic region or not. If not, the mass-fluxes are corrected by adding a quadratic friction law between layers whose coefficient is computed so that the corrected cell average is as near as possible of the boundary of the hyperbolicity region.

Finally, some numerical test have been performed to asses the efficiency of the proposed strategy.

The extension to $2 \mathrm{~d}$ models and/or high order schemes (either by means of reconstruction of states or ADER techniques) is straightforward by applying the techniques describes in [11], [2], [3], [1] ...

\section{References}

[1] M.J. Castro, M. Dumbser, C. Parés, and E.F. Toro. ADER schemes on unstructured meshes for nonconservative hyperbolic systems: applications to geophysical flows. Comp.\& Fluids 38:1731-1748, 2009 .

[2] M.J. Castro, J.J. Gallardo, and C. Parés. High order finite volume schemes based on reconstruction of states for solving hyperbolic systems with nonconservative products. Applications to shallow-water systems. Math. Comp. 75:1103-1134, 2006.

[3] M.J. Castro, E.D. Fernández, A.M.Ferreiro, J.A. García, AND C. PARÉS High order extensions of Roe schemes for two dimensional nonconservative hyperbolic systems. Jour. Sci. Comp. 39: 67114, 2009.

[4] M.J. Castro, A.M.Ferreiro, J.A. García, J.M. González, J. Macías, C. PARÉS AND M.E. VÁzQUEz On the numerical treatment of wet/dry fronts in shallow flows: applications to one-layer and twolayer systems. Math. Comp. Model. 42 (3-4): 419-439, 2005.

[5] M.J. Castro, P.G. LeFloch, M.L. Muñoz, and C. Parés. Why many theories of shock waves are necessary: Convergence error in formally path-consistent schemes. Jour. Comp. Phys. 3227: 8107-8129, 2008.

[6] M.J. Castro, J. Macías, And C. Parés. A Q-Scheme for a class of systems of coupled conservation laws with source term. Application to a two-layer 1-D shallow water system. Math. Mod. Num. Anal. 35(1):107-127, 2001. 
[7] M.J. Castro, A. Pardo, C. Parés, and E.F. Toro. On some fast well-balanced first order solvers for nonconservative systems. To appear in Math. Comp.

[8] G. Dal Maso, P.G. LeFloch, F. Murat. Definition and weak stability of nonconservative products. J. Math. Pures Appl. 74: 483548,1995 .

[9] E.D. Fernández-Nieto Aproximación Numérica de Leyes de Conservación Hiperbólicas No Homogéneas. Aplicación a las Ecuaciones de Aguas Someras. Phd. Thesis. Univ. Sevilla, 2003.

[10] J.A. García Rodríguez Paralelización de esquemas de volúmenes finitos: aplicacin a la resolución de sistemas de tipo aguas someras. Phd. Thesis. Univ. Málaga, 2005.

[11] C. PARÉs. Numerical methods for nonconservative hyperbolic systems: a theoretical framework. SIAM J. Num. Anal. 44(1): 300-321, 2006.

[12] C. Parés, M.J. Castro. On the well-balance property of Roes method for nonconservative hyperbolic systems. Applications to Shallow-Water Systems. M2AN, Vol. 38, N5, pp. 821-852, 2004.

[13] J. B. Schijf, J. C. Schonfeld. Theoretical considerations on the motion of salt and fresh water. In Proc. of the Minn. Int. Hydraulics Conv., 321-333. Joint meeting IAHR and Hyd. Div. ASCE., Sept. 1953.

[14] I. Toumi. A weak formulation of Roe approximate Riemann solver. J. Comp. Phys. 102(2): 360-373, 1992. 